\title{
Column chromatography as a useful step in purification of diatom pigments*
}

\author{
Wiktor Tokarek, Stanisław Listwan, Joanna Pagacz, Piotr Leśniak and Dariusz Latowski ${ }^{\bowtie}$ \\ Department of Plant Physiology and Biochemistry, Faculty of Biochemistry, Biophysics and Biotechnology, Jagiellonian University, \\ Kraków, Poland
}

\begin{abstract}
Fucoxanthin, diadinoxanthin and diatoxanthin are carotenoids found in brown algae and most other heterokonts. These pigments are involved in photosynthetic and photoprotective reactions, and they have many potential health benefits. They can be extracted from diatom Phaeodactylum tricornutum by sonication, extraction with chloroform:methanol and preparative thin layer chromatography. We assessed the utility of an additional column chromatography step in purification of these pigments. This novel addition to the isolation protocol increased the purity of fucoxanthin and allowed for concentration of diadinoxanthin and diatoxanthin before HPLC separation. The enhanced protocol is useful for obtaining high purity pigments for biochemical studies.
\end{abstract}

Key words: column chromatography, Phaeodactylum tricornutum, fucoxanthin, diadinoxanthin, diatoxanthin, thin layer chromatography

Received: 20 March, 2016; revised: 09 May, 2016; accepted: 03 June, 2016; available on-line: 02 August, 2016

\section{INTRODUCTION}

Fucoxanthin $(\mathrm{Fx})$, diadinoxanthin (Ddx) and diatoxanthin (Dtx) (Fig. 1A) are carotenoids found in a large group of marine algae but are also present in some fresh-water photoautotrophs. Fx and Ddx are both synthesized from neoxanthin, which is at a branching point in the carotenoid biosynthetic pathway from $\beta$-carotene (Dambek et al., 2012). Dtx is a result of an enzymatic de-epoxidation of Ddx (Latowski et al., 2011).

Fx is the main accessory pigment of the brown-algae and most other heterokonts, such as sea- and fresh-water diatoms. It is present in the light-harvesting complex, called fucoxanthin-chlorophyll protein, absorbs visible light in the $480-560 \mathrm{~nm}$ region and transfers the excitation energy to chlorophyll (Chl) molecules with one of the highest efficiencies among known carotenoids ( $\mathrm{Pa}$ pagiannakis et al., 2005). Recently, a lot of research has been focused on Fx, as it exhibits many properties that can be beneficial to the human health. First of all, it is a potent antioxidant, able to efficiently quench the singlet oxygen and scavenge free radicals. These properties are attributed to the presence of an unusual allenic bond $(C=C=C)$ (Sachindra et al., 2007). Moreover, Fx can act as an antioxidant under anoxic conditions, which is very rare among carotenoids (Nomura et al., 1997). The beneficial health properties of Fx include anti-cancer (induction of apoptosis and cell cycle arrest in cancer cells), anti-obesity (induction of uncoupling protein-1 in white adipose tissue), anti-photoageing and antidiabetic activities (summarized by D'Orazio et al., 2012; Mikami \&
Hosokawa, 2013; Kumar et al., 2013; Moghadamtousi et al., 2014; Miyashita \& Hosokawa, 2015). Furthermore, it is capable of inhibiting the inflammatory response (Choi et al., 2015).

$\mathrm{Ddx}$ is involved in a photoprotective diadinoxanthin cycle, which is typical for some groups of photosynthetic algae (diatoms, phaeophytes, dinophytes, and haptophytes) and protects these organisms from an oxidative stress mainly generated by high light intensity (Latowski et al., 2011). Under high light conditions, Ddx molecules are de-epoxidized to yield Dtx molecules, which have one more conjugated double bond than Ddx. This conversion is responsible for avoiding damage caused by intensive illumination (Lavaud et al., 2002). Dtx has been shown to suppress the expression of pro-inflammatory cytokines in murine cells (Konishi et al., 2008), and both of these pigments are considered as constituents of sunscreens (Johnsen et al., 2008).

While there are numerous publications describing the beneficial effects of Fx, the Ddx and Dtx have not been studied extensively, especially in animal or human cells, mainly because they are present is the source material in low amounts (Carreto \& Cataggio, 1976) and their purification can be problematic.

In this work, we introduced an additional column chromatography step to an already established method for extraction of diatom pigments (Sadura, 2014). We assessed the utility of this novel pre-separation step in small-scale isolation procedure of all mentioned pigments. We used diatoms, which we found as the only known source allowing the simultaneous preparative isolation of Fx, Ddx and Dtx (Cohen, 1999; Pfeil et al., 2014)

\section{MATERIALS AND METHODS}

Diatom cultures. Phaeodactylum tricornutum strain 1055/1 was obtained from the Culture Collection of Algae and Protozoa (CCAP; UK). The diatoms were grown for $5-8$ days at $12^{\circ} \mathrm{C}$ in flasks containing a sterile Guillard's f/2 medium (Guillard, 1975) without added silicate. The light intensity was $48 \mu \mathrm{mol} \mathrm{m} \mathrm{m}^{-2} \mathrm{~s}^{-1}$ (as measured with Skye PAR Quantum) and the light/dark regime was 14/10 hours. Before pigment extraction,

e-mail: dariusz.latowski@uj.edu.pl

*Presented at the 43rd Winter School of the Faculty of Biochemistry, Biophysics and Biotechnology of the Jagiellonian University "Biomolecules: from structure to function", February 16-20, 2016, Zakopane, Poland.

Abbreviations: Ddx, diadinoxanthin; Dtx, diatoxanthin; Chl, chlorophyll; Fx, fucoxanthin; HP-TLC, high-performance thin layer chromatography; $\mathrm{RT}$, retention time 
A $\mathrm{Fx}$

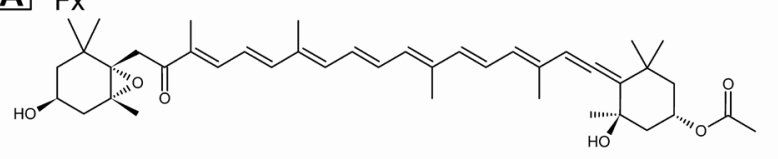

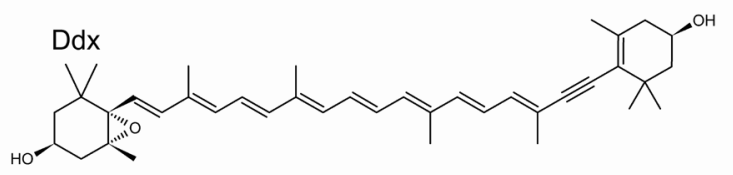<smiles>[R]C1(C)CC(O)CC(C)=C1/C=C/C(C)=C/C=C/C(C)=C/C=C/C=C(C)/C=C/C=C(C)C#CC1=C(C)CC(O)CC1(C)C</smiles>

B

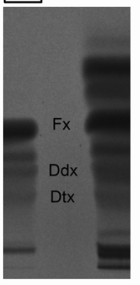

C
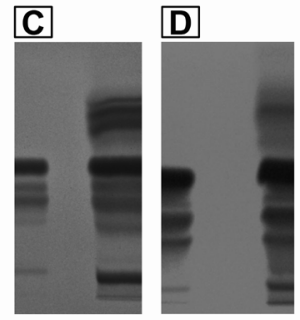

E

F

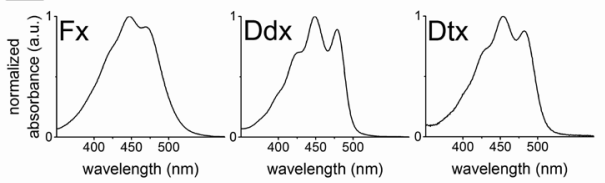

Figure 1. Pigment characteristics.

(A) chemical formulas of Fx, Ddx and Dtx. (B-E) fragments of representative HP-TLC plates after pigment separation. The left side of each plate shows the separation of pigments, when column pre-separation step was used and the mobile phase contained petroleum ether (B and $\mathbf{D})$ or extraction gasoline (C and $\mathbf{E})$. The right side of each plate shows the separation when no column pre-separation was used. The pigments were obtained from diatom cultures that were either kept in the darkness (panels B and C) or illuminated (panels D and E) before centrifugation. (F) representative spectra of Fx, Ddx and Dtx, recorded in acetone, using pigments isolated when column pre-separation with a solvent containing extraction gasoline was utilized. The $\mathrm{R}_{\mathrm{f}}$ values are $0.44,0.32$ and 0.23 for Fx, Ddx and Dtx, respectively.

the flasks were kept in darkness for at least 1 hour (in the case of Fx and Ddx isolation) or were subjected to intense illumination $\left(1550 \mu \mathrm{mol} \mathrm{m} \mathrm{m}^{-2} \mathrm{~s}^{-1}\right)$ for 1.5 hours, while stirring (in the case of Dtx isolation).

Pigment extraction. Grown cultures were centrifuged $\left(4^{\circ} \mathrm{C}, 10 \mathrm{~min}, 4000 \mathrm{rcf}\right)$, the pellet was suspended in one volume of the extraction buffer $(50 \mathrm{mM}$ phosphate buffer, $\mathrm{pH}$ 8.0, $300 \mathrm{mM} \mathrm{NaCl}$ ) and sonicated (Sonics Vibra-Cell VC505, 10 minutes, 15s/10s on/off, amplitude $35 \%$ ). This was followed by centrifugation $\left(4^{\circ} \mathrm{C}, 10 \mathrm{~min}\right.$, $16900 \mathrm{rcf}$ ) and the supernatant was collected in a separate tube. The extraction solvent used was a mixture of chloroform:methanol 1:2. In the first extraction step, $1 \mathrm{~mL}$ and $0.7 \mathrm{~mL}$ of the extraction solvent was added to the pellet and supernatant, respectively. Both samples were mixed thoroughly and centrifuged, as described above. The organic phases from both samples were collected, and the aqueous phase from the supernatant sample was discarded. In the second extraction step, $1 \mathrm{~mL}$ of the extraction solvent was added to both, the pellet and supernatant sample. The samples were handled as described above (Sadura, 2014). For each purification procedure, a sample containing $1.5 \mu \mathrm{mol}$ of $\mathrm{Chl}$ a was prepared.

HP-TLC separation. The collected organic phases, containing chlorophylls and carotenoids, were evaporated using nitrogen gas and dissolved in the HP-TLC solvent (methanol:water: $\mathrm{NH}_{3}$ 90:10:0.0012). The pigment solution was transferred to the HP-TLC plate (silica gel 60 RP-8 $\mathrm{F}_{254} \mathrm{~s}$; Merck Millipore) using Linomat 5 (Camag, Switzerland). After separation, the pigments were scraped, extracted with acetone (Sadura, 2014), separated from silica by centrifugation, and stored at $-20^{\circ} \mathrm{C}$.

Column chromatography. An additional separation step was added between pigment extraction and HP-TLC separation. Two solvents were used in this step - petroleum ether (b.p. $60-80^{\circ} \mathrm{C}$ ):acetone:n-propanol 60:40:3 (Aitzetmüller et al., 1968) and extraction gasoline $\left(\mathrm{n}=7-8\right.$, b.p. $90^{\circ} \mathrm{C}$ ):acetone:n-propanol 60:40:3. Glass column (length $-20 \mathrm{~cm}$, internal diameter - $10 \mathrm{~mm}$ ) was filled with neutral calcinated $\mathrm{Al}_{2} \mathrm{O}_{3}$ in a given solvent (Aitzetmüller et al., 1968). The extracted pigments were evaporated, dissolved in the smallest amount of a given solvent and loaded onto the column. The separation was light protected and conducted at room temperature. The orange, carotenoid-containing fraction was collected, evaporated, dissolved in the HP-TLC solvent and separated on a HP-TLC plate, as described above.

Spectrophotometric analysis. Pigment spectra were measured in acetone, using the V-650 spectrophotometer (Jasco, Japan), and compared to the representative spectra presented by Jeffrey and coworkers (1997). Fx, Ddx and Dtx concentrations were assessed using a molar extinction coefficients equal to $109000 \mathrm{~mol}^{-1} \mathrm{~cm}^{-1}$ (Haugan \& Liaaen-Jensen, 1989), $130000 \mathrm{~mol}^{-1} \mathrm{~cm}^{-1}$ (Johansen et al., 1974) and $119000 \mathrm{~mol}^{-1} \mathrm{~cm}^{-1}$ (Johansen et al., 1974), respectively. Chl a's concentration was measured in $90 \%$ acetone according to Ritchie (2006).

HPLC analysis. HPLC setup consisted of an Agilent Technologies (USA) 1260 Quaternary Pump VL, 1260 Diode Array Detector VL and Nucleosil 300-5 C18 $250 \times 4 \mathrm{~mm}$ analytical column (Chromatographie-Service $\mathrm{GmbH}$, Germany). The following solvents were used: extraction $(90 \%, \mathrm{v} / \mathrm{v}$ [9:1 methanol:ammonium acetate $0.2 \mathrm{M}], 10 \%$, v/v ethyl acetate), A (85:15 methanol:ammonium acetate $0.5 \mathrm{M}), \mathrm{B}$ (9:1 acetonitrile:water), C (ethyl acetate). Ammonium acetate solution was prepared and filtered right before use. All other solvents were of an HPLC grade. Before separation, the acetone sample was evaporated, dissolved in the extraction solvent and loaded onto a column. The separation was carried out

Table 1. Solvent gradients used in HPLC analysis (Kraay et al., 1992)

\begin{tabular}{llll}
\hline Time (min) & Solvent A (\%) & Solvent B (\%) & Solvent C (\%) \\
\hline 0 & 60 & 40 & 0 \\
\hline 2 & 0 & 100 & 0 \\
\hline 7 & 0 & 80 & 20 \\
\hline 17 & 0 & 50 & 50 \\
\hline 21 & 0 & 30 & 70 \\
\hline 28.5 & 0 & 30 & 70 \\
\hline 29.5 & 0 & 100 & 0 \\
\hline 30.5 & 60 & 40 & 0 \\
\hline 35 & 60 & 40 & 0 \\
\hline
\end{tabular}



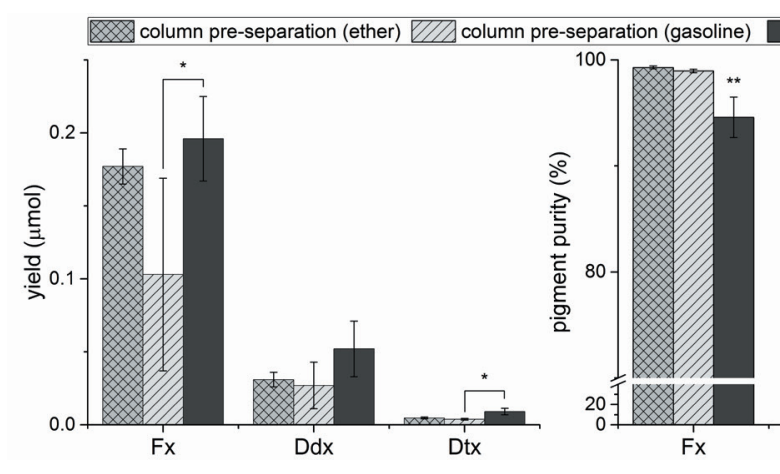

no column pre-separation

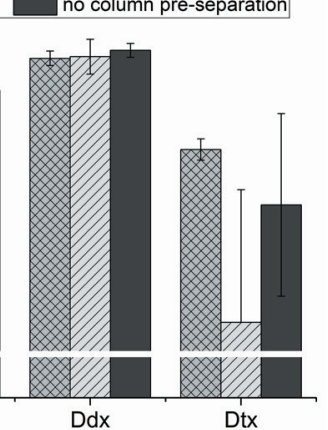

Figure 2. Yield and purity.

Purification procedure yield (from half of an HP-TLC plate; left) and the purity of obtained pigments (right). The column pre-separation step was conducted using a solvent containing either petroleum ether ("ether") or extraction gasoline ("gasoline"). * $p<0.04$ by ANOVA. **Kruskall-Wallis rank sum test determined that there is a significant difference between results in this group $(p=0.0127)$; Wilcoxon rank sum test was used to determine that there is no significant difference between results obtained using column pre-separation step $(p=0.1)$ in the said group.

as described by Kraay et al. (1992), using the flow rate

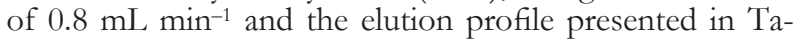
ble 1 . The wavelengths used for detection were 430, 440 and $480 \mathrm{~nm}$. The peaks were identified by their spectra (Jeffrey et al., 1997) and retention times (Kraay et al., 1992).

Statistical analysis. The experiments were repeated at least three times (with or without the column pre-separation step). The data were analyzed with the $\mathrm{R}$ software, version 3.2.4 ( $\mathrm{R}$ Core Team, 2016). The data concerning the purity of pigments and procedure yield were grouped in three categories, reflecting the employed procedures (no column pre-separation and pre-separation using solvent containing either petroleum ether or extraction gasoline). If the data in groups passed the Barlett test of homogeneity of variances, ANOVA and Tukey multiple comparisons of means were used to determine which means are significantly different from each other. If the data failed the Barlett test, then Kruskal-Wallis rank sum test was used to check if there is a difference between groups. Wilcoxon rank sum test was then used to determine which groups are not significantly different from each other.

\section{RESULTS AND DISCUSSION}

\section{Pigment pre-separation}

As a result of the additional column chromatography step, the separation of carotenoids from Chls was obtained. This may be especially important, as free Chl is a photosensitizer and can, upon illumination, lead to the generation of reactive oxygen species (Marder et al., 1998; Krieger-Liszkay, 2005). This in turn may contribute to the lower pigment quality and purification yield. By introducing this purification step, we decreased the time when carotenoids are mixed with Chls and hence lowered the possibility of carotenoids being damaged by the photodynamic effect caused by free Chls. The separations where petroleum ether was used were completed slightly faster than those where the extraction gasoline was used. This may be attributed to the lower density of petroleum ether, as it consists of shorter hydrocarbon chains.

\section{Spectral quality of purified pigments}

The representative spectra of Fx, Ddx and Dtx are presented in Fig. 1F. The obtained spectra were consistent with those presented by Jeffrey and coworkers (1997). The additional column separation step did not cause a significant improvement in respect to spectral quality (general shape, peak positions and ratios), no matter which solvent was used. Furthermore, no isomerization was detected by this analysis. In some spectra, minuscule peaks originating from Chl were present (not shown). They were present more frequently when no column pre-separation step was employed. This supports the idea that additional column chromatography step increases the purity of the isolated pigments, while the spectral quality of the main constituent remains comparable.

\section{Purification procedure yield}

In the case of Fx and Ddx, when column pre-separation step was utilized, the solvent containing petroleum ether gave better results than the one containing the extraction gasoline. In these instances, the yield was higher in comparison to the other solvent and the yield variability was much lower. When Dtx is considered, the results were similar, as the yield was higher in the case of the solvent containing petroleum ether (Fig. 2). The highest overall yield was obtained for Fx, which is present in P. tricornutum cells in the largest amounts (Carreto \& Cataggio, 1976). Pre-separation with column chromatography did not lead to the increase of the purification yield. It can be attributed to the fact that the additional purification procedure causes greater material losses. On the other hand, column pre-separation step eliminates the Chls from the extracts and allows for the separation of more concentrated samples on HP-TLC.

\section{Pigment purity}

The highest pigment purities obtained were above 99\%, 99\% and 93\% for Fx, Ddx and Dtx, respectively. In the case of all tested pigments, the pigment purity (assessed by HPLC analysis) did not differ significantly between the two solvents used in the column chromatography step (Fig. 2). When this additional step was omitted, the purity of Fx was significantly lower, while the purity of Ddx and Dtx remained comparable to the purity obtained using the pre-separation step. This can be explained by the fact that $\mathrm{Chl}$ bands are closely located to the Fx band on the HP-TLC plate (Fig. 1B-E). This hinders the obtaining of Fx. Moreover, while the HP-TLC separation of the sample is still developing, $\mathrm{Fx}$ remains in contact with Chls for a longer time than Ddx and Dtx in the sample that has not been pre-separated, which are characterized by a lower retardation factor in the system used in this study. It can lead to photodamage of the Fx molecules when the HP-TLC plate is accidentally illuminated. This can explain the contaminations detected by the HPLC analysis with Chls and carotenoid isomers (Fig. 3). In the case of Ddx, we observed the 


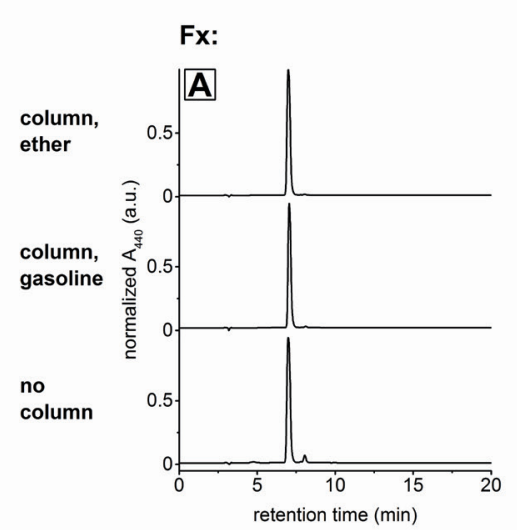

after
HPLC
Ddx:
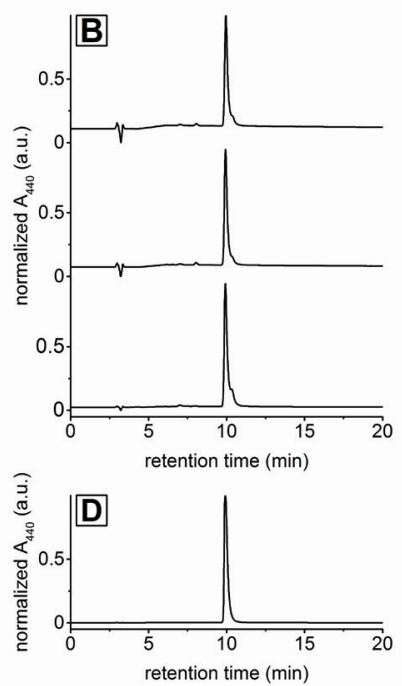

Dtx:
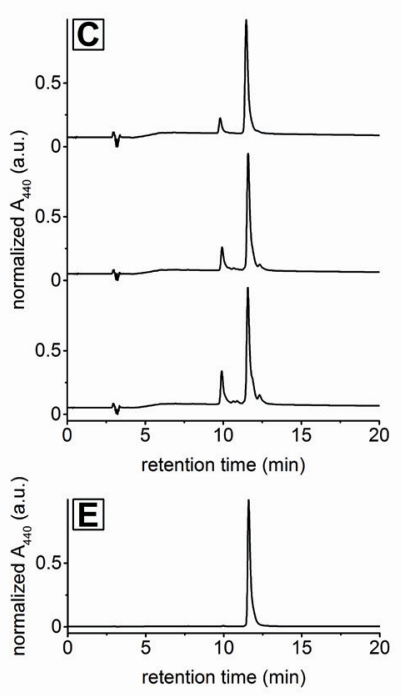

Figure 3. Representative chromatograms of Fx, Ddx and Dtx purified using different methods.

(A) Fx (RT $7.0 \mathrm{~min}$ ). (B) Ddx (RT $9.9 \mathrm{~min}$ ). (C) Dtx (RT $11.5 \mathrm{~min}$ ). The pigments were isolated using a column chromatography step with mobile phase containing petroleum ether (top) or extraction gasoline (middle), or without this additional step (bottom). (D-E) fractions collected after the preparative HPLC separation of $\operatorname{Ddx}(\mathbf{D})$ and $\operatorname{Dtx}(\mathbf{E})$ samples that were pre-concentrated using the column chromatography step. Presented chromatograms show the first 20 out of 35 minutes of separation, according to gradient presented in Table 1. The detected contaminations are Chls (RT $4.84 \mathrm{~min}$ ) and other carotenoids (RT 8.02, 10.35 and $12.34 \mathrm{~min}$ ). Dtx samples are contaminated with Ddx, which can be eliminated by preparative HPLC separation. Chromatograms were normalized to 1 at the peak maximum.

co-elution of another compound, most probably a diadinochrome (Jeffrey et al., 1997). These compounds are formed when Ddx epoxide groups are converted to furanoid groups during a purification procedure (LiaaenJensen, 1989). This co-eluting compound was consistently present in all analyzed samples of Ddx, which indicates that the group rearrangement is not caused by the reagents used in the column chromatography $\left(\mathrm{Al}_{2} \mathrm{O}_{3}\right.$, solvents).

The Dtx samples were almost always contaminated with small amounts of Ddx, which can be attributed to their close proximity on a HP-TLC plate.

\section{Further purification using HPLC}

In order to remove contaminations, the samples of Ddx and Dtx were subjected to further purification, using HPLC (the same method as described above). The collected fractions of Ddx (RT 9.6-10.2 min) and Dtx (RT 11.3-12 min) were analyzed once again by HPLC. The results demonstrated that the purity of Ddx exceeded $99.7 \%$ and that of Dtx reached $99.5 \%$, with room for improvement.

We also developed a shorter version of the protocol for preparative HPLC separation of Fx, Ddx and Dtx, which comprises of the same solvents and takes only 20 minutes (Fig. 3).

\section{CONCLUSIONS}

The additional pre-separation step, utilizing the column chromatography, significantly enhances the purity of Fx and lowers its variability. The pre-separation step does not influence the purity of Ddx or Dtx, which can be further purified, employing preparative HPLC, to the purity of at least $99.5 \%$. The column pre-separation step is an easy and cheap way to concentrate the samples before HPLC separation, which lowers the overall cost of the isolation procedure. While this step decreases the overall yield, it can limit the potential photodynamic effect from Chl, as the carotenoids and Chls are separated earlier in the process. We conclude that adding a column pre-separation step can be useful for small-scale laboratory preparations of $\mathrm{Fx}$ and it allows the inexpensive concentration of Ddx and Dtx before further purification and analysis. The improved purification protocol can be used for obtaining material for further studies, concerning the biochemical and biophysical properties of Fx, Ddx and Dtx, as well as the elucidation of molecular mechanisms underlying the beneficial health effects of Fx, Ddx and Dtx. It is especially important for Ddx and Dtx, which are not well studied. We trust that the method developed in this work will advance the research focused on these pigments.

\section{Conflict of interests}

The Authors declare no conflict of interests.

\section{Acknowledgements}

We thank Bartosz Pluciński, MSc, for his help with statistical analysis.

Faculty of Biochemistry, Biophysics and Biotechnology of Jagiellonian University is a partner of the Leading National Research Center (KNOW) supported by the Ministry of Science and Higher Education. This work was supported by project No. 2011/01/M/NZ1/01170 funded by the National Science Center, Poland.

\section{REFERENCES}

Aitzetmüller K, Svec WA, Katz JJ, Strain HH (1968) Structure and chemical identity of diadinoxanthin and the principal xanthophyll of Euglena. Chem Commun 1: 32-33. http://dx.doi.org/10.1039/ C19680000032.

Carreto JI, Cataggio JA (1976) Variations in pigment contents of the diatom Phaeodactylum tricornutum during growth. Marine Biology 36: 105-112. http://dx.doi.org/10.1007/BF00388433. 
Choi JH, Kim NH, Kim SJ, Lee HJ, Kim S (2015) Fucoxanthin inhibits the inflammation response in paw edema model through suppressing MAPKs, Akt, and NFxB. J Biochem Mol Toxicol 30: 111119. http://dx.doi.org/10.1002/jbt.21769.

Cohen Z (1999) Chemicals from Microalgae. Cohen Z ed, pp 149-160. Taylor \& Francis Ltd. ISBN 0-7484-0515-1.

Dambek M, Eilers U, Breitenbach J, Steiger S, Büchel C, Sandmann G (2012) Biosynthesis of fucoxanthin and diadinoxanthin and function of initial pathway genes in Phaeodactylum tricornutum. $J$ Exp Bot 63: 5607-5612. http://dx.doi.org/10.1093/jxb/ers211.

D’Orazio N, Gemello E, Gammone MA, de Girolamo M, Ficoneri C, Riccioni G (2012) Fucoxantin: a treasure from the sea. Mar Drugs 10: 604-616. http://dx.doi.org/10.3390/md10030604.

Guillard RRL (1975) Culture of phytoplankton for feeding marine invertebrates. In Culture of marine invertebrate animals. Smith WL, Chanley MH eds, pp 26-60. Plenum Press, New York, USA. http:// dx.doi.org/10.1007/978-1-4615-8714-9_3.

Haugan JA, Liaaen-Jensen S (1989) Improved isolation procedure for fucoxanthin. Phytochemistry 28: 2797-2798. http://dx.doi. org/10.1016/S0031-9422(00)98091-9.

Jeffrey SW, Mantoura RFC, Bjørnland T (1997) Data for identification of $47 \mathrm{key}$ phytoplankton pigments. In Phytoplankton pigments in oceanography: guidelines to modern methods. Jeffrey SW, Mantoura RFC, Wright SW eds, pp 493-560. UNESCO Publishing.

Johansen JE, Svec WA, Liaaen-Jensen S, Haxo FT (1974) Carotenoids of the Dinophyceae. Pbytochemistry 13: 2261-2271. http://dx.doi. org/10.1016/0031-9422(74)85038-7.

Johnsen G, Lysaa PA, Aamodt K (2014) Sunscreen Compositions Comprising Carotenoids. Promar AS, assignee. Patent US 8834855 B2.

Konishi I, Hosokawa M, Sashima T, Maoka T, Miyashita K (2008) Suppressive effects of alloxanthin and diatoxanthin from Haloynthia roretzi on LPS-induced expression of pro-inflammatory genes in RAW264.7 cells. J Oleo Sci 57: 181-189. http://dx.doi.org/10.5650/ jos.57.181.

Kraay GW, Zapata M, Veldhuis MJW (1992) Separation of chlorophylls c1, c2, and c3 of marine phytoplankton by reversedphase-C18-high-performance liquid chromatography. J Phycol 28: 708-712. http://dx.doi.org/10.1111/j.0022-3646.1992.00708.x.

Krieger-Liszkay A (2005) Singlet oxygen production in photosynthesis. J Exp Bot 56: 337-346. http://dx.doi.org/10.1093/jxb/erh237.

Kumar SR, Hosokawa M, Miyashita K (2013) Fucoxanthin: a marine carotenoid exerting anti-cancer effects by affecting multiple mechanisms. Mar Drugs 11: 5130-5514. http://dx.doi.org/10.3390/ md11125130.

Latowski D, Kuczyńska P, Strzałka K (2011) Xanthophyll cycle - a mechanism protecting plants against oxidative stress. Redox Report 16: 78-90. http://dx.doi.org/10.1179/174329211X13020951739938.

Lavaud J, Rousseau B, van Gorkom HJ, Etienne AL (2002) Influence of the diadinoxanthin pool size on photoprotection in the marine planktonic diatom Phaeodactylum tricornutum. Plant Physiol 129: 13981406. http://dx.doi.org/10.1104/pp.002014.

Liaaen-Jensen S (1989) Artifacts of natural carotenoids - unintended carotenoid synthesis. In Carotenoids: chemistry and biology. Krinsky NI, Mathews-Roth MM, Taylor RF eds, pp 149-165. Plenum Press, New York, USA. http://dx.doi.org/10.1007/978-1-4613-0849-2_9.

Marder JB, Droppa M, Casp V, Raskin VI, Horváth G (1998) Lightindependent thermoluminescence from thylakoids of greening barley leaves. Evidence for involvement of oxygen radicals and free chlorophyll. Physiologia Plantarum 104: 713-719. http://dx.doi. org/10.1034/j.1399-3054.1998.1040428.x.

Mikami K, Hosokawa M (2013) Biosynthetic pathway and health benefits of fucoxanthin, an algae-specific xanthophyll in brown seaweeds. Int J Mol Sci 14: 13763-13781. http://dx.doi.org/10.3390/ ijms140713763.

Miyashita K, Hosokawa M (2015) The beneficial health effects of fucoxanthin. In Genomics, proteomics and metabolomics in nutraceuticals and functional foods, second edition. Bagchi D, Swaroop A, Bagchi M eds, pp 122-134. John Wiley \& Sons, Ltd, Chichester, UK. http://dx.doi. org/10.1002/9781118930458.ch10.

Moghadamtousi SZ, Karimian H, Khanabdali R, Razavi M, Firoozinia M, Zandi K, Kadir HA (2014) Anticancer and antitumor potential of fucoidan and fucoxanthin, two main metabolites isolated from brown algae. The Scientific World Journal, Article ID 768323. http:// dx.doi.org/10.1155/2014/768323.

Nomura T, Kikuchi M, Kubodera A, Kawakami Y (1997) Proton-donative antioxidant activity of fucoxanthin with 1,1-diphenyl-2-picrylhydrazyl (DPPH). Biochem Mol Biol Int 42: 361-370. http://dx.doi. org/10.1080/15216549700202761.

Papagiannakis E, van Stokkum IHM, Fey H, Büchel C, van Grondelle $\mathrm{R}$ (2005) Spectroscopic characterization of the excitation energy transfer in the fucoxanthin - chlorophyll protein of diatoms. Photosynth Res 86: 241-250. http://dx.doi.org/10.1007/s11120-005-10038.

Pfeil BE, Schoefs B, Spetea C (2014) Function and evolution of channels and transporters in photosynthetic membranes. Cell Mol Life Sci 71: 979-998. http://dx.doi.org/ 10.1007/s00018-013-1412-3.

$\mathrm{R}$ Core Team (2016) R: A language and environment for statistical computing. $R$ Foundation for Statistical Computing, Vienna, Austria. URL https://www.R-project.org/.

Ritchie RJ (2006) Consistent sets of spectrophotometric chlorophyll equations for acetone, methanol and ethanol solvents. Photosynth Res 89: 27-41. http://dx.doi.org/10.1007/s11120-006-9065-9.

Sachindra NM, Sato E, Maeda H, Hosokawa M, Niwano Y, Kohno M, Miyashita K (2007) Radical scavenging and singlet oxygen quenching activity of marine carotenoid fucoxanthin and its metabolites. J Agric Food Chem 55: 8516-8522. http://dx.doi.org/10.1021/ jf071848a.

Sadura I (2014) Isolation and purification of diadinoxanthin cycle pigments and their influence on molecular dynamic of artificial membranes. Master's Thesis, Faculty of Chemistry, Jagiellonian University in Krakón, Poland (in Polish). 